\title{
An alternative technique to reduce time, cost and human effort during natural or manufactured disasters
}

\author{
Samaher Al-Janabi ${ }^{1}$, Ayad Alkaim ${ }^{2}$, Ahmed Rahem ${ }^{1}$ \\ ${ }^{1}$ Department of Computer Science, Faculty of Science for Women (SCIW), University of Babylon, Babylon, Iraq \\ ${ }^{2}$ Department of Chemistry, Faculty of Science for Women (SCIW), University of Babylon, Babylon, Iraq
}

\begin{tabular}{l} 
Article Info \\
\hline Article history: \\
Received Mar 17, 2021 \\
Revised May 4, 2021 \\
Accepted Aug 26, 2021 \\
\hline
\end{tabular}

Keywords:

APM platform

GPS module

Internet of things

LoRa modem

NetLogo simulation

\begin{abstract}
The world suffers from a very large number of missing persons, ranging from about 250,000 to about one million people for various reasons in recent years. Therefore, the process of finding and tracing the missing persons as a result of a plane crash, fire or explosion in a particular area is a humanitarian and religious and national duty, and is one of the most important issues in our country. So, the idea of using a drone to find missing people was invested. We have designed system that collects real-time data and analyzes in a smart way and utilizes global positioning system (GPS) to locate people and track their impact. The idea of this work has been developed and implemented. They represent it in the form of a triangular problem, which included: First, how to locate the missing persons and send video broadcasts to a calculator or mobile device remotely. Analyze the data collected in real time, and send a report identifying the safe path that can be taken to reach the missing persons. The second stage is collection the data in the master computer and analysis it, while the final stage determines the coordinates of the location to the missing persons and the best possible way to reach of them.
\end{abstract}

This is an open access article under the CC BY-SA license.

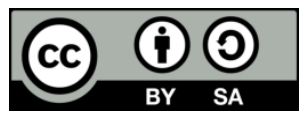

\section{Corresponding Author:}

Samaher Al-Janabi

Department of Computer Science, Faculty of Science for Women (SCIW), University of Babylon

Hillah, Babylon, Iraq

Email: samaher@itnet.uobabylon.edu.iq

\section{INTRODUCTION}

Drones have become more popular and widespread used. They have been applied in many areas such as used by courier companies in the delivery of mail services as well as solar power stations [1], use drones to collect solar energy in solar panels as well as the use of geographic personnel drones to explore the topography. Many traditional people use drones in photography and have also been used in 3D mapping [2], [3] drones are also used to search and rescue people in the event of disaster or war or when someone is lost in the wilderness or forest [4]-[25]. Victims are often injured and unconscious. Therefore, it is important to take advantage of electronic and information systems to obtain and locate these missing persons so that we can find and track. Drone as a term has many different meanings. When we talk about a drone as a device in which mechanical precision is associated with electronic, the first thing envisioned by our mind is the missiles or aircraft that are controlled remotely [4], [26]. It can be defined by an aircraft or a drone that can travel independently, without human control or out of sight.

An unmanned aerial vehicle (UAV) is an aircraft without a human pilot onboard. UAVs are a component of an unmanned aircraft system (UAS), which include a UAV, a ground-based controller [19]. The flight of UAVs may operate with various degrees of autonomy, either under remote control by a human operator or autonomously by onboard computers, (e.g. flown by a pilot at a ground control station) or can fly 
autonomously based on pre-programmed flight plans or more complex dynamic automation systems. UAVs are currently used for a number of missions [20], including tracing. For the purposes of this article, a UAV is defined as being capable of controlled, sustained level flight and powered by a jet or reciprocating engine. The acronym UAV has been expanded in some cases to unmanned aircraft vehicle system (UAVS). The federal aviation administration (FAA) has adopted the acronym UAS to reflect the fact that these complex systems include ground stations and other elements besides the actual air vehicles. UAVs are descended from target drones and remotely piloted vehicles (RPVs) employed by the military forces of many countries in the decades immediately after World War I [21].

The idea of this work has been developed and implemented in order to address a number of real problems experienced by society as a result of natural disasters or wars, specifically helping rescue teams to find missing persons. It represents a trigonometric problem that included: First, how to identify missing persons and send an audio video to a remote computer or mobile device for people's situations and whereabouts. Analyze data collected in real time in intelligent ways and send a report that identifies the safe path to be taken to reach the missing persons as shown in Figure 1.

\section{Internet not a variable Problem}

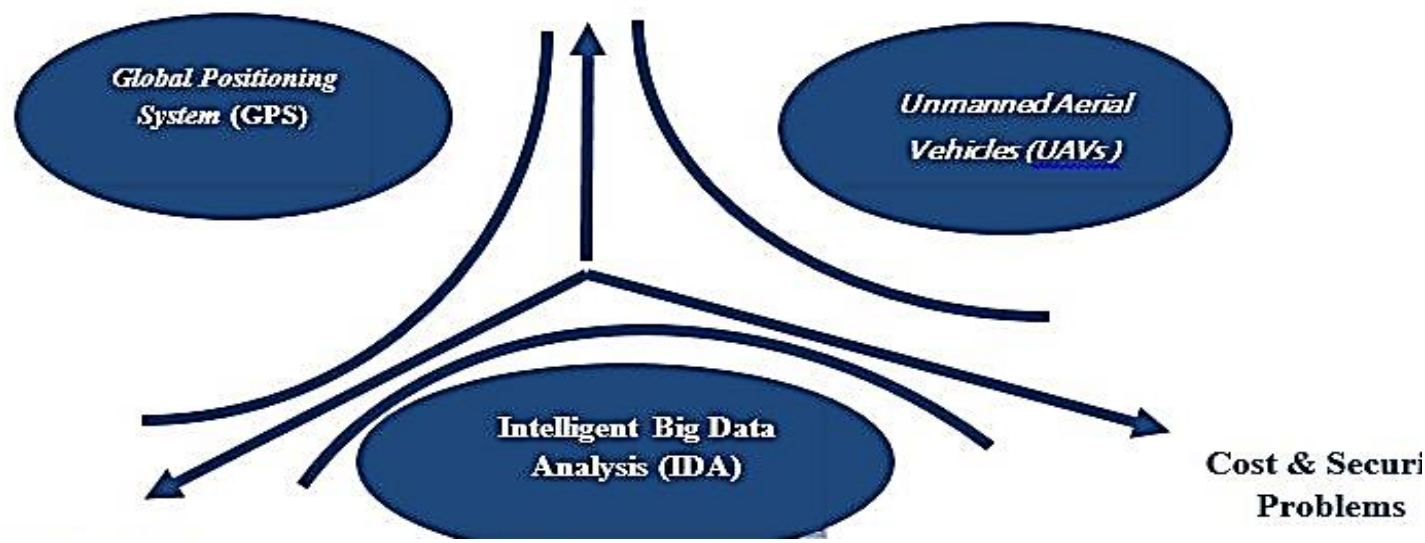

Figure 1. Relationship among the main three challenges

\section{MATERIAL AND TOOLS}

\subsection{UAV}

A UAV is a small, remote-controlled aircraft, sometimes called a "drone" that can cover large areas. Also do not carry any human pilot or passengers. Drones are completely or partially independent, but are often remotely controlled by people. In the recent past, drones were mostly associated with the military, where they were initially used in targeted anti-aircraft practice, and intelligence gathering. Today, UAVs are used in a wide range of civilian applications ranging from search and rescue, traffic control, weather monitoring, firefighting, photography, video photography, agriculture and even delivery services.

The first remote-controlled drone was used in World War I. Hayat et al. [8] In 1918, the United States military (US military) developed an experimental Kettering Bug, a non-bombed aircraft that subsequently increased the use of drones in a wider range of applications. Drones are being used in oil and gas facilities for security, surveillance, emergency response and infrastructure inspection. In seaports, drones can conduct applications such as mapping, surveying, operational supervision, port control, traffic control and also in mining operations, drones can be used to manage stocks, waste dams, inspections and more. The use cases of the drone according to an analysis carried out in more than 100 different countries and more than 80 different manufacturers and the results are shown in Figure 2. 


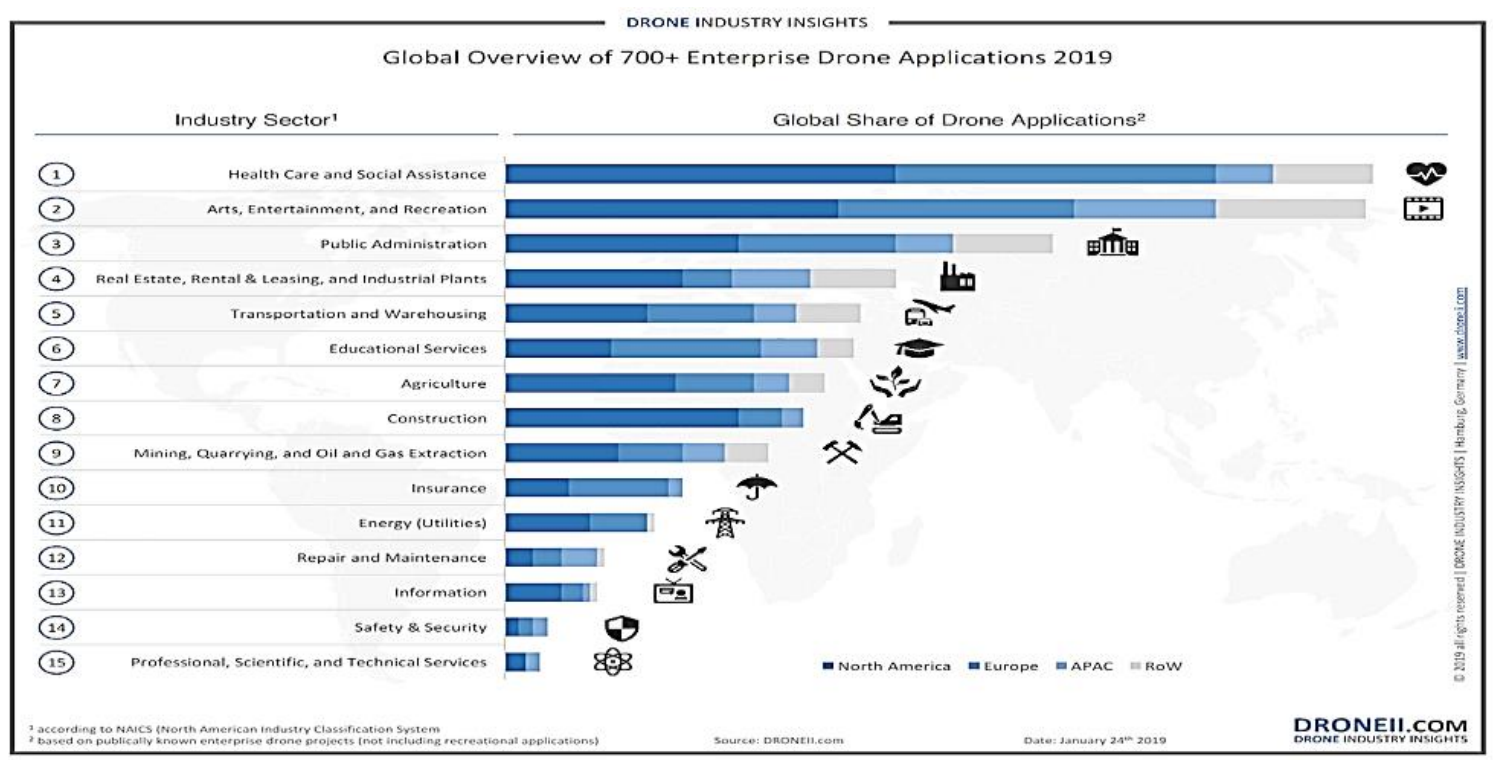

Figure 2. Results of the report [16]

\subsection{GPS module}

An updated "NEO-6M", global positioning system module (GPS module) that can be used with the "Ardupilot mega V2 (APM)". This GPS module uses the latest technology to provide the best possible location information, allowing for improved performance using Ardupilot or any other multi-motor control system. It also includes "Honeywell's" latest high-precision digital magnetic gauge (HMC5883L), which provides a convenient way to mount the compass away from interference sources that may be located near the multi-stage flight controller [9], [14].

The system relies on knowledge of the distance between two points in time is well known, the first point is a device that sends a signal in a very precise, well-defined time (GPS device associated with 514), the second point is the location of a device that receives such a reference (mobile device or a computer) as in Figure 3, and then calculate the time spent by the signal to reach the receiver (1).

Distance between transmitters and receivers $=$ time $\times$ signal flow speed

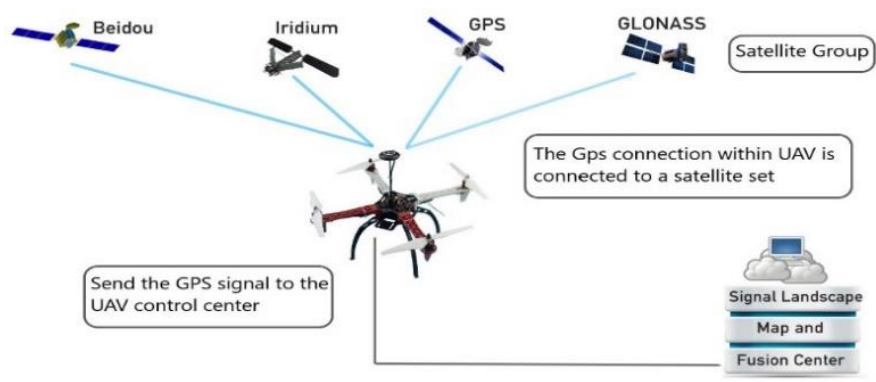

Figure 3. Demonstrates the work of GPS with UAV

\subsection{NetLogo simulation}

It is a free modelling environment capable of simulating complex situations such as natural and social phenomena. Suitable for research and education, it can be defined as a simple enough environment to enable students and researchers to create their own Java programming language models. They are particularly suitable for forming complex systems that evolve over time. Designers can give instructions to hundreds or thousands of independent agents, all of whom work concurrently. This makes it possible to explore the links between individual micro-level behaviors and macro-level patterns that arise from their interactions. It allows 
users to simulate different environments using agents, and to explore their behaviors under different circumstances [9]. Agents move through a network of "corrections" using several rules to adjust their path towards the target and the possibility of passing any obstacles they encounter in their own way in programmable ways. All agents can interact with each other and perform multiple tasks concurrently [10].

\subsection{Control system}

A control system is a device or group of devices that manages, directs, or regulates the behavior of other devices or systems. Industrial control systems are used in industrial production to control equipment or machinery. There are two common categories of control systems, open loop control system, and close loop control system. When, we determine the period of rotation according to the degree of dirty clothes, this means that the user does not need to check whether the clothes clean or not because the results are known according to the specified time. In closed-loop control systems, the current output is taken into account and corrections are made based on observations. For example, human interaction with the computer where it is a reciprocal process between human development of the computer and the improvement of its performance on the one hand, and accelerate the performance of operations, and save time and effort by the computer on the other [11], [12]. The closed-loop system is also called the feedback control system [13].

\subsection{Target detection}

Target identification means the ability to identify targets or other objects based on data obtained from sensors based on smart algorithms or methods that analyze that data, as well as to detect the target and identify its location. [12], [18].

\section{THE UAV DESIGN STAGES}

In this section, the construction of the UAV model includes several stages, the first stage is the construction phase of the drone structure, the second stage is uploading a map of the specific area in its natural state and build database of the most important entities a variable in that region and its locations, then build software system to collection the data in real time and analysis it. Finally, the process of matching the real data from the system with the original database and generating reports identifying the location of missing persons. The stages can be illustrated by the algorithm 1 called finding and tracking missing person through drone (FTMP), show the main stage of the proposal structure with all details while, Figure 4 present the main activates will achieved in this study.

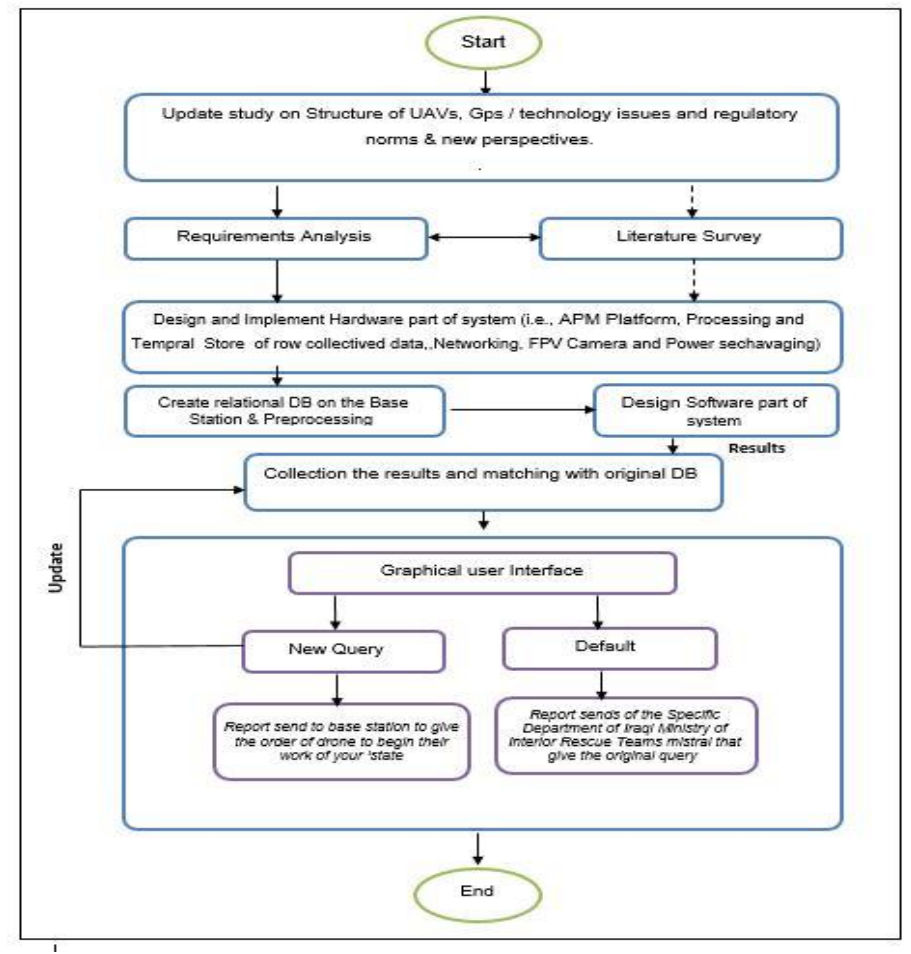

Figure 4. Flow chart of research work activities 
Input: Raw Data in Real Times.

Output: Optimal strategy for Finding and Tracking of Missing Persons by UAV

Step 1: Building the hardware (Drone structure) part

Step 2: Collect raw data from the Location points and pass it into base station.

Step3: Preprocessing that including upload of the map represent the location contain the missing persons.

Step 4: Building finder and tracker software have four procedures,

Call these procedures sequentially (i.e. Shade, Get NonShaded, offline computation, drone's behavior)

Step 5: Forward the process data in step 2 into finder and tracker software.

Then apply the Marching with the original databased to generation

Reports shown the location of Missing Persons.

Step 6: Integration the user with system through user interface.

Do for each new query

IF this query as default (i.e. come from responsible side in the country)

Being drone Service

Using Drone Service according to the following structure

\begin{tabular}{|l|l|l|l|l|}
\hline Type of user $(E, C, D)$ & ID of user & Service code & Subservice code & Reserved \\
\hline
\end{tabular}

End FTMP

Where, the user divided into three types: E: Ambiguity person, C: Constant person, D: Dynamic person

\subsection{Design electronic system to collect data in real time and save it on master computer}

This stage include design the hardware part that complete with the structure described in first stage. In general, this stage consists of two parts: i) The input devices the information to the system in real time called (location point); ii) One device to collection the information from all the Location points this device will called (base station).

The accuracy and the type of information that can be obtained from the specific area to be detected a missing person are considered important variables that help to find this people, also it improves the performance of the search process itself. Therefore, the location points collect data from a real place of the loss persons. Each device starts work when the flight of the aircraft above the selected area to start the firstperson view (FPV) camera with imaging sensors complementary metal oxide semiconductor (CMOS) or charge coupled device (CCD) that has been connected with a video sending "ImmersionRC" for feeding video directly from this region, and after the video reception by monitoring device ground (mobile phone) or computer.

While the process of collecting information from devices location points requesting a network of wide area network (WAN) type design based on a special type of devices sending called (ImmersionRC modem), and receiving data called (FPV receiver modem). These devices are characterized by being operating within Altrdad 800/900 MHz by 21 channel wireless communication (wireless channel) each channel can accommodate 254 points. Since the communication between the main node (base station) and the rest of the parts of the network will be through its own node address.

\subsection{Upload map of the area specified natural condition and build an integrated database of the most important entities in that region and their locations}

This stage is divided into two parts, the first part is loaded map of the region that has missing people, where the specified area in control plane without a pilot and specifically on the computer where he picks up a computer radio signal emitted by technology GPS, which in turn contain important information about the status satellite caller GPS as well as the current date and time. This part of the signal is necessary to determine the location. After getting a picture of a map of the affected area section 2 of this phase begins, of building a database of all components or entities that are primarily those specific environments. So, that it is suitable for data storage model in a way that avoided redundancy through correlation tables specific relations environment, as well as speed in the search query.

\subsection{Building software system consists of several steps}

This stage represents the integration between the data obtained from working environment and drone's software process. Therefore, the software at the first determine the region that drone can search it or move unit, which simulates the actual environment. In general, we will look for four procedures for the detection of missing persons, that procedures will calling sequentially as explain in algorithm 1. For more details to see how this four procedures work see [26].

\subsection{Matching process}

Is a matching process between the coordinates of the entities to the original map, which was upload in step 2 and all the new entities that have been captured by the camera of a drone. The purpose of 
conformity is explained in the following cases: i) IF each drone captures different object, it's then typical as object in the original database and it's considered as a natural condition; ii) When the drone captures the objects, and we compare then to the original database when find that it doesn't match. So, we send the coordinates of the object that doesn't have any matched object for in the database; iii) When we extract the position of the objects that the planes capture right before the process of matching as mentioned in step 1, step 2. We notice if there are more than one drone to captures the same position of the object that doesn't match any object in the database.

\section{IMPLEMENTATION}

After building the integrated airframe, in the previous chapter and identify the most important parts and components used in it. This chapter will explain the implementation and result of each step of design structure. Also, we will used NetLogo as simulation of the design model.

\subsection{Connect components of UAV structure}

The practical connection among the main components of UAV structure to achieve the goal of this paper shown in Figure 5 according to the diagram of the actor circuit. This electrical circle used the following:

\subsubsection{Accelerometer}

An electromechanical device used to measure acceleration forces such forces may be static, like the continuous force of gravity or, as is the case with many mobile devices, dynamic to sense movement or vibrations. Acceleration is the measurement of the change in velocity, or speed divided by time.

\subsubsection{GPS}

The incorporation of GPS receivers in advanced drones allows for GPS drone waypoint navigation. This is an advanced technology that allows a drone to autonomously fly to preprogrammed points. This system can instruct the drone how fast, how high, and where to fly. It can also be programmed to hover for a period of time over each waypoint. Drones are increasingly being used for surveying building construction, road maintenance, and infrastructure inspections. Agricultural applications include crop inspection, and tracking farm animals. Using GPS drone waypoint navigation, an area can be inspected at the predesignated positions. The drone controller is then able to focus his attention on the camera, and conduct the inspection. The advancement of GPS drone navigation has greatly increased their utility and range of applications. Figure 5 is showing design structure of UAV.

According to the Figure 5, we can summarization the main particle steps of connection as: i) Connecting the main structure (quadcopter frame) in the form of a signal (+), with four arms; ii) The main structure contains a panel (PCB) to connecting the drone parts in a battery (Li-po battery) to connect the power; iii) Connect motors (brushless DC motor) in each arm, and connect propellers in motors obverse; iv) Install the control of motors) electronic speed control (ESC) (on each arm, each ESC is connected with motors on the one hand and with PCB on the other. Wires ESC are also connected to the receiving device (ImmersionRC audio/video transmitter); v) Connect the camera (FPV) with (Servo Gimbal) installed in frame. The camera has three connecting wires (red, black, yellow), the red and black wire is connected with (universal battery eliminator circuit (UBEC)) which in turn are connected with the panel PCB to connect the power. The yellow wire is connected with the video transmitter (ImmersionRC) installed in the frame; vi) Install the signal receiver (MC8RB) in one of the arms of frame, which has 8 channels with antenna. Channels (1 to 5) connect with (ArduPilot APM 2.8 flight controller), and channel 7 and 8 connect with servo gimbal which controls the FPV camera; vii) Install flight controller in the frame, to be part of the main structure. Contains flight controller (APM) 8 pins, connected (1 to 5) pins with channels (1 to 5) respectively in the receiver (MC8RB), also contains 3 pins connect with ESC. It contains several ports such as (GPS, OSD, 3DR, PM), and universal serial bus (USB) type-C port to connect the device with the personal computer (PC); viii) Is installed (GPS) in the frame and connect it with flight controller APM via an outlet GPS port; ix) To start drone aircraft, the Li-po battery is connected to the board PCB, also as connect (APM) to the PC and work (ARM2) by software (Mission Planner). Remote control of the aircraft is done by (Microzone MC8B); $\mathrm{x}$ ) The (ImmersionRC) device sends video captured by FPV camera to the receiving device video signal (FPV Receiver), which in turn is associated with the mobile device, where we use an Android software called (Go FPV app) to watch the video. To illustrate these steps, see Figure 6. 


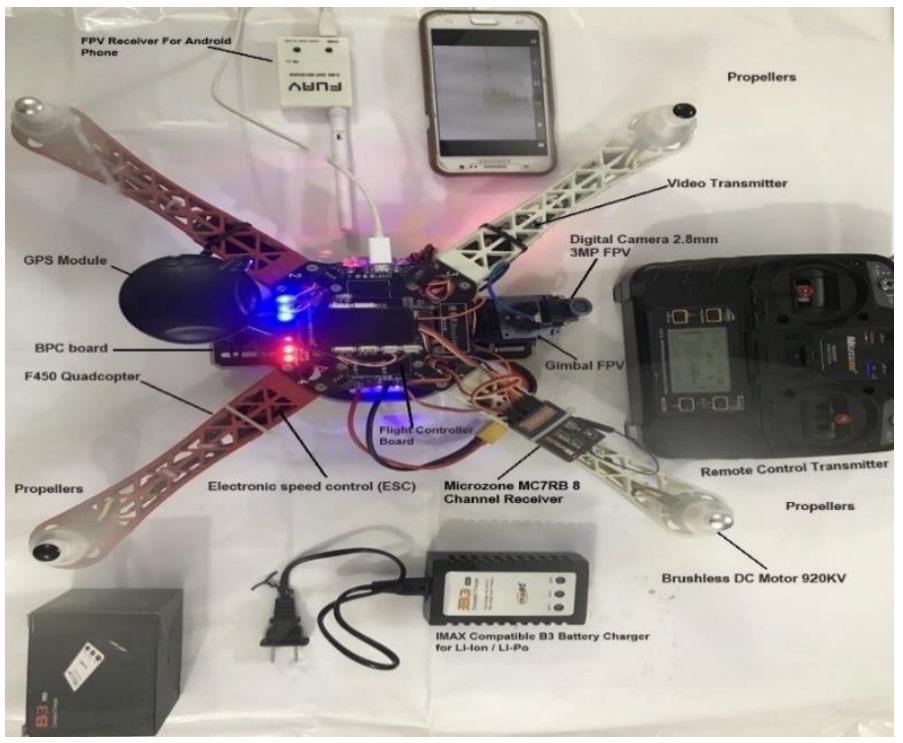

Figure 5. Design structure of UAV
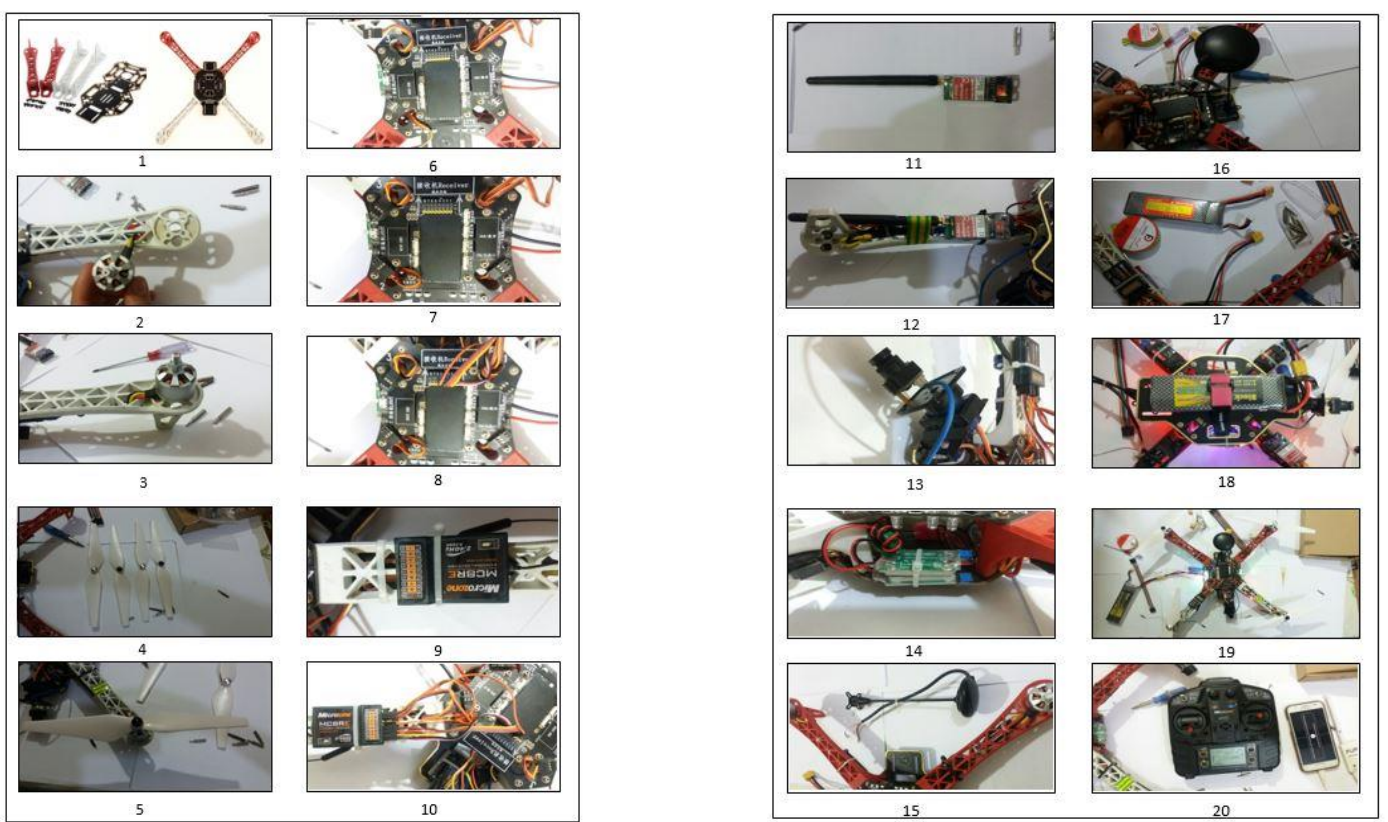

Figure 6. Connect main tools

\subsection{Build database for all objects (elements) in that specific area}

The integrated system was implemented using a NetLogo version 6.0.2, The model was created to represent the movement of unmanned aircraft over a defined area. Figure 7 shown the main screen of system.

The model uses three breeds of agents: pads, which just signal through their color whether they're available for landing (green), available only to empty drones (blue), or occupied by a drone (red); They have a capacity, which is the maximum number of packets that can be uploaded to the platform at the same time. The main interface contains a toolkit: i) Total-targets: this tool represents total number of peoples expected missing in a specific area; ii) Total-finders: a tool used to determine the route of drone planes in the specified area; iii) Vision: this tool represents a vision which we mean the vision of the drone within the specified region; iv) Setup: this tool is used to adjust the work of the simulation before starting the implementation or configuration; v) Go: tool used to begin the work of drone. 


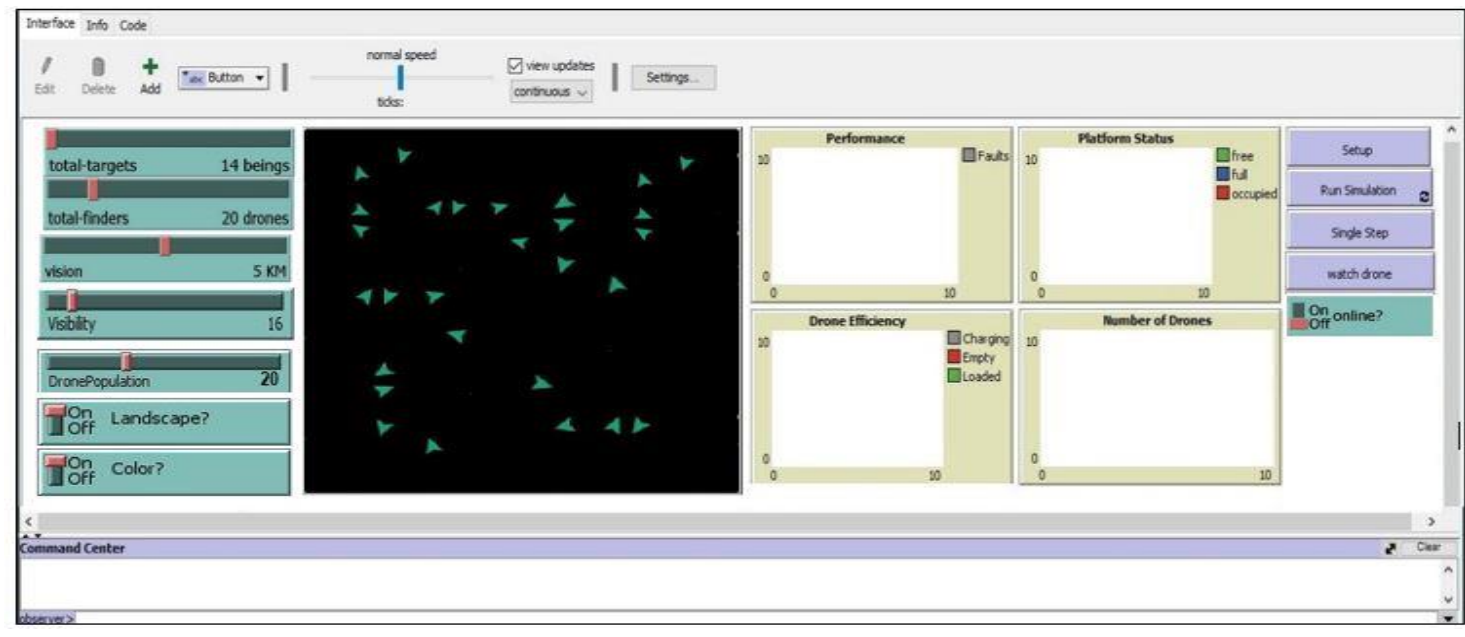

Figure 7. Model of UAVs to finding and tracking of missing persons

\section{CASE STUDY}

To explain the main steps will all details, we will assume have 14 persons lost in specifies area. Therefor where, order give to find them the system in two parts will start: i) Step 1: upload to the system the map of the area lost the person on it, and build database for the more popular object in that area. As explained a Figure 8. This is a step to discover the path for missing persons to find the shortest path and reach them. The step uses a specific area map, including buildings, corridors, driving routes and water. Passengers select the building randomly as a destination, search and follow the shortest route to get there; ii) Step 2: deployment the drones design through initial steps of this paper on the specific region after determined the initial coordinate $(\mathrm{X}, \mathrm{Y})$ for each drone as explain in Table 1; iii) Step 3: determined the path of people through using the camera of UAV and tracking them by GPS. After loading the map, the process of determining the path of people to reach them according to the coordinates of the drone corresponding to the coordinates of the person as shown in Figure 9; In figure 9, the track of the person no. 3 which was found according to the coordinates of certain facts. red route is a route of the person and movements in the region, and the yellow path represents the path of the drone data has been established according to the coordinates of these persons are shown in the Table 2; iv) Step 4: matching between the original data set of area and the dataset capture through drones, then generated the final report have specific coordinated for each person determined its location.

Table 1. Initial coordinates for each drone

\begin{tabular}{cccc}
\hline \#Drone & X-coordinates & Y-coordinates & Time \\
\hline 0 & 9.69 & 13.82 & $3: 00 \mathrm{PM}$ \\
1 & 14.87 & 6.56 & $3: 01 \mathrm{PM}$ \\
2 & 1.90 & 14.51 & $3: 01 \mathrm{PM}$ \\
3 & 14.99 & 5.17 & $3: 02 \mathrm{PM}$ \\
4 & 7.07 & 14.78 & $3: 12 \mathrm{PM}$ \\
5 & 14.56 & 7.92 & $3: 13 \mathrm{PM}$ \\
6 & 1.41 & 14.33 & $3: 15 \mathrm{PM}$ \\
7 & 12.07 & 12.07 & $3: 15 \mathrm{PM}$ \\
8 & 10.44 & 13.3 & $3: 20 \mathrm{PM}$ \\
9 & 14.99 & 5.34 & $3: 22 \mathrm{PM}$ \\
10 & 8.78 & 14.27 & $3: 22 \mathrm{PM}$ \\
11 & 14.90 & 3.60 & $3: 30 \mathrm{PM}$ \\
12 & 10.29 & 13.48 & $3: 30 \mathrm{PM}$ \\
13 & 14.96 & 5.87 & $3: 33 \mathrm{PM}$ \\
14 & 13.09 & 10.87 & $3: 33 \mathrm{PM}$ \\
15 & 4.47 & 14.98 & $3: 38 \mathrm{PM}$ \\
16 & 12.31 & 11.81 & $3: 40 \mathrm{PM}$ \\
17 & 12.43 & 3.60 & $3: 42 \mathrm{PM}$ \\
18 & 8.74 & 14.27 & $3: 45 \mathrm{PM}$ \\
19 & 8.74 & 14.27 & $3: 49 \mathrm{PM}$ \\
\hline
\end{tabular}




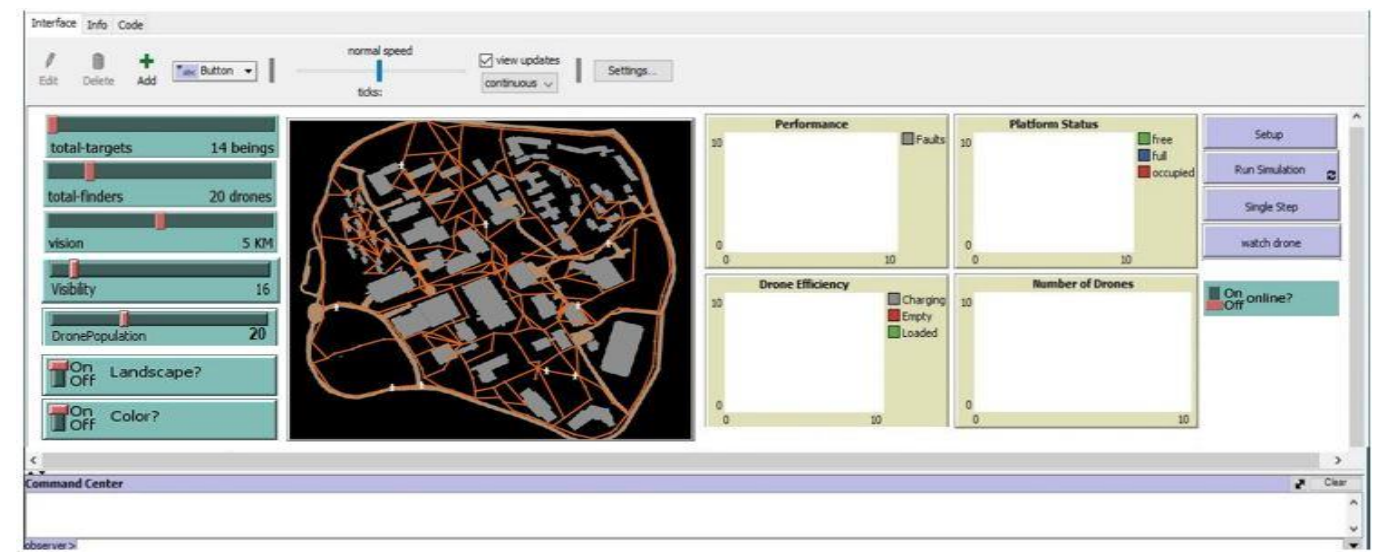

Figure 8. Upload a map for a specific area

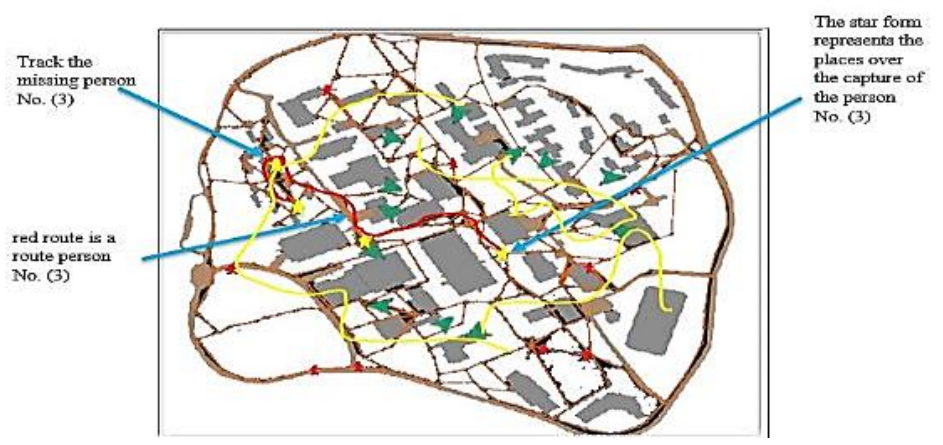

Figure 9. Upload map of specific location

Table 2. Dataset finds through drone

\begin{tabular}{|c|c|c|c|c|c|c|c|c|}
\hline \multirow{2}{*}{ \#Drone } & \multirow{2}{*}{$\mathrm{X}$-coordinates } & \multirow{2}{*}{ Y- coordinates } & \multirow{2}{*}{ count } & \multirow{2}{*}{ Time } & \multicolumn{3}{|c|}{ Person } & \multirow{2}{*}{ Time } \\
\hline & & & & & $\mathrm{X}-\mathrm{cc}$ & dinates & Y- coordinates & \\
\hline 7 & 12.07 & 12.07 & 1 & 3:00 PM & P6 & 12.07 & 12.07 & 3:01 PM \\
\hline 1 & 14.87 & 6.56 & 1 & 3:01 PM & P7 & 14.87 & 6.56 & 3:03 PM \\
\hline \multirow{2}{*}{10} & 8.78 & 14.27 & \multirow{2}{*}{2} & \multirow{2}{*}{ 3:01 PM } & P1 & 8.78 & 14.27 & 3:07 PM \\
\hline & 7.04 & 13.01 & & & $\mathrm{P} 2$ & 7.04 & 13.01 & 3:10 PM \\
\hline 9 & 14.99 & 5.34 & 1 & 3:01 PM & P3 & 14.99 & 5.34 & 3:15 PM \\
\hline 14 & 14.87 & 6.56 & 1 & 3:15 PM & P7 & 14.87 & 6.56 & 3:16 PM \\
\hline 13 & 14.99 & 5.34 & 1 & 3:17 PM & P3 & 14.99 & 5.34 & 3:19 PM \\
\hline 15 & 4.47 & 14.98 & 1 & 3:20 PM & P4 & 4.47 & 14.98 & $3: 22$ PM \\
\hline 6 & 1.41 & 14.33 & 1 & 3:22 PM & P8 & 1.41 & 14.33 & $3: 23 \mathrm{PM}$ \\
\hline 4 & 8.74 & 14.27 & 1 & $3: 24$ PM & P5 & 8.74 & 14.27 & 3:25 PM \\
\hline 16 & 14.99 & 5.34 & 1 & 3:31 PM & P3 & 14.99 & 5.34 & 3:32 PM \\
\hline 19 & 8.74 & 14.27 & 1 & 3:40 PM & P5 & 8.74 & 14.27 & 3:44 PM \\
\hline 0 & 8.78 & 14.27 & 1 & 3:41 PM & P1 & 8.78 & 14.27 & $3: 42$ PM \\
\hline 11 & 1.41 & 14.33 & 1 & 3:41 PM & P8 & 1.41 & 14.33 & 3:44 PM \\
\hline 17 & 14.87 & 6.56 & 1 & 3:42 PM & P7 & 14.87 & 6.56 & 3:47 PM \\
\hline 8 & 4.47 & 14.98 & 1 & 3:50 PM & P4 & 4.47 & 14.98 & 3:52 PM \\
\hline 3 & 7.04 & 13.01 & 1 & 3:50 PM & $\mathrm{P} 2$ & 7.04 & 13.01 & 3:54 PM \\
\hline 12 & 14.99 & 5.34 & 1 & 3:55 PM & P3 & 14.99 & 5.34 & 3:57 PM \\
\hline 18 & 4.47 & 14.98 & 1 & 3:55 PM & P4 & 4.47 & 14.98 & 3:56 PM \\
\hline 2 & 7.04 & 13.01 & 1 & 3:55 PM & $\mathrm{P} 2$ & 7.04 & 13.01 & 3:57 PM \\
\hline 5 & 1.41 & 14.33 & 1 & 3:57 PM & P8 & 1.41 & 14.33 & 3:58 PM \\
\hline
\end{tabular}

\section{CONCLUSIONS AND FUTURE WORKS}

We can summarize the main points conclusion from this work by the following: design a pragmatic drone structure to find and track missing persons in a specific environment using principal analysis intelligent data (IDA) and GPS. Design and implementation of an integrated electronic circuit interfacing circuit (i.e. APM 2.8 ArduPilot), since "APM 2.8 ArduPilot" is not simply used, five supporting parts are real time clock, battery $3000 \mathrm{~m} \mathrm{A/L,} \mathrm{GPS} \mathrm{model,} \mathrm{(wireless} \mathrm{audio/video} \mathrm{TX/RX} \mathrm{(for} \mathrm{live} \mathrm{video),} \mathrm{remote} \mathrm{control} \mathrm{transmitter).}$ 
Using global positioning system to develop the efficiency of performance in the field of security in Iraq. This is done by building an integrated system in terms of physical entities $\mathrm{H} / \mathrm{W}$, and $\mathrm{S} / \mathrm{W}$ saves time, effort and cost and addresses a real security problem in the country. Using "APM 2.8 ArduPilot" architecture to collect real data as a first step to start knowledge discovery from databases.

In the future, we can use the design structure in the following points: finding the locations of multiple targets in a flight and using reinforcement learning to get the location of wireless device and trace it. An optimized application will be developed to improve the system in order to make it more efficient in route tracing and drone interaction. Focus on the integration of two action cameras with mutual coverage in stereo mode, which will make it possible to increase the efficiency of imaging during one flight. The use of UAV networks in combination with the cellular network wireless sensor network (WSN), and show promising future technology for applications in disaster management.

\section{REFERENCES}

[1] E. Vattapparamban, I. Güvenç, A. I. Yurekli, K. Akkaya, and S. Uluağaç, "Drones for smart cities: Issues in cybersecurity, privacy, and public safety," in 2016 International Wireless Communications and Mobile Computing Conference, IWCMC 2016, Sep. 2016, pp. 216-221, doi: 10.1109/IWCMC.2016.7577060.

[2] F. Nex and F. Remondino, "UAV for 3D mapping applications: A review," Applied Geomatics, vol. 6, no. 1, pp. 1-15, Mar. 2014, doi: 10.1007/s12518-013-0120-x.

[3] S. Al-Janabi, A. F. Alkaim, and Z. Adel, "An Innovative synthesis of deep learning techniques (DCapsNet \& DCOM) for generation electrical renewable energy from wind energy," Soft Computing, vol. 24, no. 14, pp. 10943-10962, Jul. 2020, doi: 10.1007/s00500-020-04905-9.

[4] M. A. Ma'Sum et al., "Simulation of intelligent Unmanned Aerial Vehicle (UAV) for military surveillance," in 2013 International Conference on Advanced Computer Science and Information Systems, ICACSIS 2013, Sep. 2013, pp. 161-166, doi: 10.1109/ICACSIS.2013.6761569.

[5] S. N. Zenk, S. A. Matthews, A. N. Kraft, and K. K. Jones, "How many days of global positioning system (GPS) monitoring do you need to measure activity space environments in health research?," Health and Place, vol. 51, pp. 52-60, May 2018, doi: 10.1016/j.healthplace.2018.02.004.

[6] L. Liu, R. Moulic, and D. Shea, "Cloud service portal for mobile device management," in Proceedings - IEEE International Conference on E-Business Engineering, ICEBE 2010, Nov. 2010, pp. 474-478, doi: 10.1109/ICEBE.2010.102.

[7] E. Vartiainen and K. Väänänen-Vainio-Mattila, "User experience of mobile photo sharing in the cloud," in Proceedings of the 9th International Conference on Mobile and Ubiquitous Multimedia - MUM '10, 2010, pp. 1-10, doi: 10.1145/1899475.1899479.

[8] S. Hayat, E. Yanmaz, and R. Muzaffar, "Survey on Unmanned Aerial Vehicle Networks for Civil Applications: A Communications Viewpoint," IEEE Communications Surveys and Tutorials, vol. 18, no. 4, pp. 2624-2661, 2016, doi: 10.1109/COMST.2016.2560343.

[9] S. Al-Janabi and A. F. Alkaim, "A nifty collaborative analysis to predicting a novel tool (DRFLLS) for missing values estimation," Soft Computing, vol. 24, no. 1, pp. 555-569, Jan. 2020, doi: 10.1007/s00500-019-03972-x.

[10] K. Wackwitz, "The drone applications report 2019," Droneii.com Drone Industry Insight, 2019. https://www.droneii.com/droneapplications-2019\#comments.

[11] A. Tubadji, V. Angelis, and P. Nijkamp, "Micro-Cultural Preferences and Macro-Percolation of New Ideas: A NetLogo Simulation," Journal of the Knowledge Economy, vol. 10, no. 1, pp. 168-185, Mar. 2019, doi: 10.1007/s13132-017-0446-4.

[12] S. Tisue and U. Wilensky, "Netlogo: A simple environment for modeling complexity," in Conference on Complex Systems, 2004, pp. 1-10, [Online]. Available: http://ccl.sesp.northwestern.edu/papers/netlogo-iccs2004.pdf.

[13] O. Špinka, Š. Kroupa, and Z. Hanzálek, “Control system for unmanned aerial vehicles," in IEEE International Conference on Industrial Informatics (INDIN), Jul. 2007, vol. 1, pp. 455-460, doi: 10.1109/INDIN.2007.4384800.

[14] B. Tebrean, S. Crisan, C. Muresan, and T. E. Crisan, "Low cost command and control system for automated infusion devices," in IFMBE Proceedings, vol. 59, 2017, pp. 81-84.

[15] S. Al-Janabi, I. Al-Shourbaji, M. Shojafar, and M. Abdelhag, "Mobile Cloud Computing: Challenges and Future Research Directions," in Proceedings - International Conference on Developments in eSystems Engineering, DeSE, Jun. 2018, pp. 62-67, doi: 10.1109/DeSE.2017.21.

[16] L. Kamelia, E. A. D. Hamidi, W. Darmalaksana, and A. Nugraha, "Real-Time Online Attendance System Based on Fingerprint and GPS in the Smartphone," in Proceeding of 2018 4th International Conference on Wireless and Telematics, ICWT 2018 , Jul. 2018, pp. 1-4, doi: 10.1109/ICWT.2018.8527837.

[17] I. Bekkerman and J. Tabrikian, "Target detection and localization using MIMO radars and sonars," IEEE Transactions on Signal Processing, vol. 54, no. 10, pp. 3873-3883, Oct. 2006, doi: 10.1109/TSP.2006.879267.

[18] A. Nagaty, C. Thibault, M. Seto, M. Trentini, and H. Li, "Construction, modeling and control of an autonomous Unmanned Aerial Vehicle for target localization," in 2013 IEEE International Conference on Robotics and Biomimetics, ROBIO 2013, Dec. 2013, pp. 1544-1549, doi: 10.1109/ROBIO.2013.6739686.

[19] M. Korki, N. D. Shankar, R. N. Shah, S. M. Waseem, and S. Hodges, "Automatic Fault Detection of Power Lines using Unmanned Aerial Vehicle (UAV)," in 2019 1st International Conference on Unmanned Vehicle Systems-Oman, UVS 2019, Feb. 2019, pp. 1-6, doi: 10.1109/UVS.2019.8658283.

[20] C. Ramirez-Atencia and D. Camacho, "Extending QGroundControl for Automated Mission Planning of UAVs," Sensors, vol. 18, no. 7 , p. 2339, Jul. 2018, doi: 10.3390/s18072339.

[21] E. Akturk and A. O. Altunel, "Accuracy assesment of a low-cost UAV derived digital elevation model (DEM) in a highly broken and vegetated terrain," Measurement: Journal of the International Measurement Confederation, vol. 136, pp. 382-386, Mar. 2019, doi: 10.1016/j.measurement.2018.12.101.

[22] P. B. Rich, "Cinema, drone warfare and the framing of counter-terrorism," Defense and Security Analysis, vol. 34, no. 2, pp. 144160, Apr. 2018, doi: 10.1080/14751798.2018.1478185.

[23] X. Zhang, D. Zhao, D. Lyu, and H. Ma, "Poster: AutoCup: A platform for automatically creating aerial panoramic map with multi-UAVs," in MobiSys 2019 - Proceedings of the 17th Annual International Conference on Mobile Systems, Applications, and Services, Jun. 2019, p. 530, doi: 10.1145/3307334.3328609. 
[24] A. F. Alkaim and S. Al_Janabi, "Multi Objectives Optimization to Gas Flaring Reduction from Oil Production," in Lecture Notes in Networks and Systems, vol. 81, 2020, pp. 117-139.

[25] "Waspote development Wiki.” http://www.libelium.com/development/waspmote/documentation/waspmote.

[26] M. Lodeiro-Santiago, I. Santos-González, and P. Caballero-Gil, "UAV-based rescue system for emergency situations," in Lecture Notes in Computer Science (including subseries Lecture Notes in Artificial Intelligence and Lecture Notes in Bioinformatics), vol. 10070 LNCS, 2016, pp. 163-173.

\section{BIOGRAPHIES OF AUTHORS}

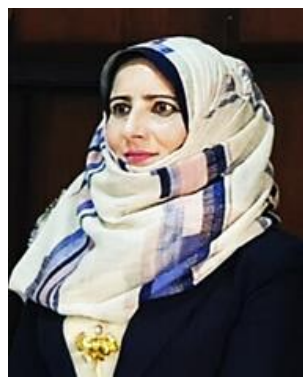

Samaher Al-Janabi (iD S SC P received her BSc, MSc, and PhD degrees in Computer Science from Science College, University of Babylon, Iraq. Through her years of study, she specialized in the design, implementation, performance of measurement and intelligent analysis of huge/bigdata, databases. She is a Lecturer in the Departments of Software, Computer and Information Network respectively. Her research interests span topics concerning intelligent data analysis, knowledge discovery in database, soft computing techniques and applications, data \& graph mining techniques (i.e., clustering, classification, predication, optimization and association rules), cooperative computing (cluster, grid, and cloud), internet of things, mobile services \& applications. she has published well over sixtynine scientific papers and authored three books; one on new trends of KDD toward intelligent data analysis, and one book on soft computing techniques, while, the third intelligent miner of huge medical database. She is a one from five women winner the L'Oreal-UNESCO for Women in Science Levant and Egypt Regional Fellowships 2014. She has get patent 2018 for your' research under title" Smart System to create Optimal Higher Education Environment in Iraq using IDA and IoTs" and the other patent for research under title "A novel method to design smart station to prediction the concentrations of air pollution (PM2.5, PM10, NO2, CO, O3, SO2) based on intelligent big data analysis" in 2021. While the third patent for your researcher under title "An innovative model of (UAV-FTMP) captures the signals of smart watches and smartphones to finding and tracking of missing persons in specific area based on intelligent data analysis and global positioning system" in 2021. She is a one from four persons on the ACM-W Rising Star Award Selection Committee for years 2020 and 2021. She is a reviewer of several local and international journals. She can be contacted at email: samaher@itnet.uobabylon.edu.iq.

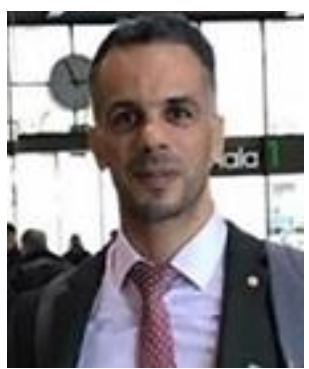

Ayad Alkaim (D) SC P professor of chemistry, is working as a researcher associate of Prof. Dr. Falah Hassan Hussein at University of Babylon since 2011; also, he is working as a coworker at the Institute of Technical Chemistry of the Leibniz University Hannover since 2012, professional activities at the Leibniz University Hannover, Germany, at the Institute of Technical Chemistry, and at Babylon University College of science for women/chemistry department. His research interests co mprise photocatalytic water and wastewater treatment, preparations of nonmaterial and studied the optical activities, Adsorption model designs, and self-cleaning surfaces. Dr. Alkaim one of the authorized of the chemical safety and security in Babylon university/Iraq, He participating in several workshops of chemical safety and security as international and national participants. He has published well over Eighty-three scientific papers. He has gotten Patent 2018 for your' research under title "Innovative method for synthesizing carbon nanotubes from natural gas" in 2018 and the other patent for research under title "A novel method to design smart station to prediction the concentrations of air pollution (PM2.5, PM10, NO2, CO, O3, SO2) based on intelligent big data analysis" in 2021. While the third patent for your researcher under title "An innovative model of (UAV-FTMP) captures the signals of smart watches and smartphones to finding and tracking of missing persons in specific area based on intelligent data analysis and global positioning system" in 2021. He is editor and a reviewer of several local and international journals. He can be contacted at email: alkaimayad@gmail.com

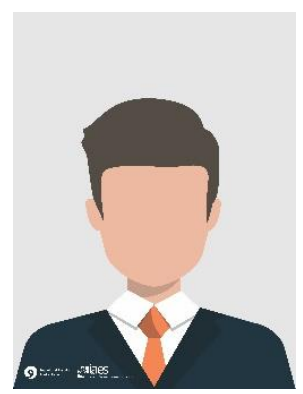

Ahmed Rahem (D) SC P is a new researcher, received the BS degree in computer science from Science College, University of Kefa, Iraq. His research interests span topics concerning graph mining techniques (i.e., clustering, classification, prediction, and optimization, and association rules). He can be contacted at email: ahmed2@gmail.com 